\title{
A basal carbon concentrating mechanism in plants?
}

\author{
Eduardo Zabaleta ${ }^{1 *}$, M. Victoria Martin ${ }^{1}$ and Hans-Peter Braun ${ }^{2}$
}

${ }^{1}$ Instituto de Investigaciones Biológicas IIB-CONICET-UNMdP, Funes 3250 3er nivel 7600 Mar del Plata, Argentina

${ }^{2}$ Institute for Plant Genetics, Leibniz Universität Hannover, Herrenhäuser Str. 2 D-30419 Hannover, Germany

\begin{abstract}
Many photosynthetic organisms have evolved inorganic carbon (Ci) concentrating mechanisms (CCMs) that increase the $\mathrm{CO}_{2}$ concentration in the vicinity of Ribulose-1,5-bisphosphate Carboxylase/Oxygenase (RubisCO). Several CCMs have been described in great detail, like Four carbon (C4) and Crassulacean acid metabolism (CAM), bicarbonate accumulation systems and capsular structures around RubisCO. These systems are believed to have evolved several times as a mechanism that to acclimate organisms to unfavourable growth conditions. Based on recent experimental evidence we propose the occurrence of another more general CCM system present in all plants. This basal CCM (bCCM) is supposed to be composed of mitochondrial carbonic anhydrases (a $\beta$-type carbonic anhydrase and the $\gamma$-type carbonic anhydrase domain of the mitochondrial NADH dehydrogense complex) and probably further unknown components. The bCCM is proposed to reduce leakage of $\mathrm{CO}_{2}$ from plant cells by allowing efficient recycling of mitochondrial $\mathrm{CO}_{2}$ for carbon fixation in chloroplasts.
\end{abstract}

*Corresponding author: Dr. Eduardo Zabaleta Instituto de Investigaciones Biológicas IIBCONICET-UNMdP, Funes 3250 3er nivel 7600 Mar del Plata, Argentina e-mail: ezabalet@mdp.edu.ar 


\section{Tables of content}

Abstract

1. Introduction

2. Types of CCMs

2.1 C4 and CAM mechanisms

2.2 CCM in Chlamydomonas

2.3 CCM in Cyanobacteria

3. Proposed basal CCM

3.1. Evidence for the basal CCM

4. Outlook

References

Abreviations C3: Three Carbon, C4: Four Carbon, CA: Carbonic Anhydrase, CAL: Carbonic Anhydrase Like, Cam: Carbonic Anhydrase Methanosarcina sp., CAM: crassulacean acid metabolism, CCM: Carbon Concentrating Mechanism, Ci: inorganic Carbon, CMS: Cytoplasmic Male Sterility, DiT: Dicarboxylic Acid Translocase, EZA: EthoxZolAmide, gcvT: cyanobacterial plant-like glycine decarboxylase, glc: cyanobacterial glycolate dehydrogenase, HCR: High Carbon Requiring, HPR: Hydroxypyruvate Reductase, LCI: Limiting-CO $\mathrm{O}_{2}$ Inducible, odc: cyanobacterial oxalate decarboxylase, PEP: PhosphoEnolPyruvate, RubisCO: Ribulose-1,5-bisphosphate Carboxylase/Oxygenase, SSU: Small SubUnit, SHMT: Serine HydroMethyl Transferase, TCA: Tricarboxylic Acid Cycle, tsr: cyanobacterial tartronic semialdehyde reductase.

Key words: Carbon concentrating mechanism, plant, green algae, mitochondria 


\section{Introduction}

Carboxylation of ribulose-1,5-bisphospate by the enzyme Ribulose-1,5-bisphospate Carboxylase/Oxygenase (RubisCO, E.C. 4.1.1.39) is the main source of organic carbon for almost all living organisms. However, due to a side activity of RubisCO, oxygenation of ribulose-1,5bisphospate also can take place. The ratio of the carboxylation and oxygenation reactions catalyzed by RubisCO depends on the $\mathrm{CO}_{2}$ and $\mathrm{O}_{2}$ concentration in the vicinity of the enzyme [1]. On a global scale, one out of four reactions catalysed by RubisCO leads to oxygenation of ribulose-1,5bisphosphate. However, oxygenation can be much higher under certain conditions, for example high temperatures or aridity dry conditions. As a consequence of the oxygenation of ribulose-1,5bisphosphate, one molecule of 3-phosphoglycerate and one of 2-phosphoglycolate are formed in chloroplasts.

Since 2-phosphoglycolate represents a potent inhibitor of photosynthesis, it has to be converted into other compounds. This mainly takes place by the photorespiration pathway: The process of photorespiration is based on this: The 2-phosphoglycolate is first dephosphorylated to glycolate in the chloroplast and transported into the peroxisome where it is oxidized to glyoxylate. This reaction is linked to hydrogen peroxide $\left(\mathrm{H}_{2} \mathrm{O}_{2}\right)$ formation, which is then detoxified by a peroxisomal catalase. Subsequently, glyoxylate is aminated into glycine which is then transported to the mitochondrion. In mitochondria, two molecules of glycine are converted into one serine by the glycine decarboxylase (E.C. 2.1.2.10) and serine hydromethyl transferase (EC 2.1.2.1-SHMT) enzymes. This conversion is linked to reduction of $\mathrm{NAD}^{+}$and liberation of $\mathrm{CO}_{2}$ and $\mathrm{NH}_{4}{ }^{+}$. Based on this conversion, three out of four carbon atoms are recovered for primary carbon metabolism. Serine formed in mitochondria is next transported back to the peroxisome where it is deaminated into hydroxypyruvate and reduced to glycerate by hydroxypyruvate reductase (EC 1.1.1.81-HPR). A second but cytosolic HPR has recently been discovered and was proposed to allow a bypass of this 
part of photorespiration pathway and/or to be important in the context of a metabolic overflow protection mechanism [2]. Finally, glycerate is phosphorylated by glycerate kinase (EC 2.7.1.31) in the chloroplast to form 3-PGA, which can be fed back into the Calvin cycle. Since the whole metabolic pathway only takes place in the light and leads to liberation of $\mathrm{CO}_{2}$ it is designated "photo-respiration". The pathway of course co-exists with classical respiration (termed "dark respiration" in plants). Due to the oxygenase activity of RubisCO, it is estimated that $\mathrm{C} 3$ plants such as wheat or rice only fix about $55 \% \mathrm{CO}_{2}$ of what they theoretically could fix if RubisCO was solely a carboxylase. However, indirect measurements of photorespiration indicate a reduction in $\mathrm{Ci}$ assimilation in the range of $25-35 \%$ under present atmospheric conditions [3].

The $\mathrm{CO}_{2}$ concentration around RubisCO is crucial for proper carbon fixation to sustain plant growth and ultimately all life on earth. During evolution, especially during periods of low $\mathrm{CO}_{2}$ in the atmosphere, different biochemical and even anatomical strategies have independently emerged to overcome limiting carbon fixation, designated as inorganic "carbon concentrating mechanisms" (CCMs).

In addition to the previously described carbon concentrating mechanisms, we here propose, based on recent experimental findings, that all eukaryotic photosynthetic organisms contain a basal CCM (bCCM). Photosynthesis is estimated to be reduced up to $20 \%$ without such a bCCM (see below). The proposed bCCM is based on the allows recycling of mitochondrial $\mathrm{CO}_{2}$ for carbon fixation in chloroplasts. Besides $\mathrm{CO}_{2}$ diffusion between mitochondria and chloroplasts, we propose an active $\mathrm{CO}_{2}$ transfer mechanism which is based on the presence of carbonic anhydrases (E.C. 4.2.1.1) and bicarbonate transporters in the two involved organelles. Carbonic anhydrases are $\mathrm{Zn}-$ metalloenzymes that catalyse the reversible hydration of carbon dioxide into bicarbonate. There are five types of enzymes $(\alpha, \beta, \gamma, \delta$ and $\varepsilon)$ which are not evolutionary related. they are seen as a case of convergent evolution. We propose that the proposed basal CCM system probably has evolved from 
endosymbionts ( $\alpha$ proteobacteria) during the process of chloroplast adaptation in the modern green eukaryotic cell. In this paper, experimental evidence for a bCCM is summarized. But first, the previously described CCM systems are briefly introduced and compared.

\section{Types of CCMs}

2.1 C4 and CAM mechanisms systems Four-carbon (C4) photosynthesis and crassulacean acid metabolism (CAM) in terrestrial higher plants were the first photosynthetic CCM to be described in detail. Both systems are based on a carbon pre-fixation step (binding of bicarbonate to phosphoenolpyruvate [PEP] by PEP carboxylase -E.C. 4.1.1.31-). The carbon pre-fixation step and the final $\mathrm{CO}_{2}$ fixation step by RubisCO are spatially (C4) or temporally (CAM) separated.

In CAM plants, which often grow at very hot and dry locations, oxaloacetate is formed by PEP carboxylation during the night when stomata are open to allow gas exchange. Oxaloacetate next is converted into malate which can be stored in the vacuole. When stomata are closed during the day preventing loss of water, malate is decarboxylated providing $\mathrm{CO}_{2}$ for final carbon fixation by the Calvin cycle [4].

In C4 plants, PEP carboxylation takes place in specialized mesophyll cells that also carry out the complete light reaction of photosynthesis. Malate or aspartate are transported from mesophyll cells to another type of leaf cell termed bundle sheath cells (Kranz anatomy). At this location the pre-fixed carbon is released for final $\mathrm{CO}_{2}$ fixation by RubisCO. Due to this process, the $\mathrm{CO}_{2}$ concentration in the bundle-sheath cells is around 10-fold higher than in normal air. Thereby, photorespiration is much reduced. At the same time, most bundle sheath cells do not carry out the complete photosynthetic light reaction [1]. 
The primary $\mathrm{CO}_{2}$ fixation step catalysed by PEP carboxylase takes place in the cytosol of mesophyll cells using $\mathrm{HCO}_{3}{ }^{-}$as a substrate. Therefore, carbon dioxide coming from the external surroundings must be rapidly hydrated by a $\beta$-type carbonic anhydrase $(\beta C A)$ and converted into $\mathrm{HCO}_{3}{ }^{-}$. Thus, carbonic anhydrase activity is mainly found in the cytosol of mesophyll cells while in C3 plants, the highest carbonic anhydrase activity is detected within the chloroplast stroma [5].

The presence of $\mathrm{C} 4$ metabolism has been observed in several important crop species such as maize, sorghum and sugar cane [6] and also in submerged aquatic plants and macroalgae such as Udotea spp. [7] or the planktonic diatom Thalassiosira weissflogii [8,9], whereas CAM-like metabolism has been found mainly in terrestrial xerophytic species such as cacti, yucca and aloe but also in many aquatic plants and brown macroalgae [4,10]. In some species of Flaveria spp. and Heliotropium spp. an intermediate "C3-C4 metabolism" takes place [11]. Half of the species of Flaveria express intermediate traits between C3-like to C4-like forms [12]. Some C3-C4 intermediate species restrict glycine decarboxylation to the bundle sheath compartment and thereby thus concentrate $\mathrm{CO}_{2}$ in this cell type only into the bundle sheath cells [11]. This process also was named 'C2 photosynthesis' because the decarboxylation of photorespiratory metabolites concentrates $\mathrm{CO}_{2}$ around RubisCO, thereby increasing photosynthetic efficiency. Some genera have only few confirmed C3-C4 intermediate species (Brassica, Alternanthera, Parthenium, Neurachne, Salsola, Cleomacease) [11]. Interestingly, Conversely, some amphibious species of Eleocharis, particularly Eleocharis vivipara, express $\mathrm{C} 4$ characteristics under terrestrial conditions and C3 characteristics under submerged aquatic conditions [13].

Three species in family Chenopodiaceae perform single-cell C4 photosynthesis without Kranz anatomy $[14,15]$. These species have two chloroplast types (dimorphic chloroplasts), which are biochemically and morphologically different. Each type is located in a distinct cytoplasmic domain within individual photosynthetic cells. Single cell C4 metabolism very much resembles C4 
metabolism in $\mathrm{C} 4$ plants with Kranz-type leaf anatomy [16]. It also is based on a $\mathrm{CO}_{2}$ pre-fixation step catalyzed by PEP carboxylase, the occurrence of separate photosynthetic compartments domains and physiological responses typical of $\mathrm{C} 4$ plants.

Photorespiration is much reduced in C4 plants compared to C3 plants. However, photorespiration is an essential metabolic process since mutants affecting enzymes of the 2phosphoglycolate metabolism, e.g. glycolate oxidase (EC 1.1.3.15) of maize, are not viable in normal air but completely rescued at high $\mathrm{CO}_{2}$ conditions. A maize goxl mutant rapidly accumulates glycolate when transferred to normal air, which greatly decrease efficiency of net carbon assimilation. The existence of such kind of mutants indicates that a functional photorespiratory pathway is essential for maize seedling development, most likely for detoxification of glycolate $[17,18]$.

2.2 CCM in Chlamydomonas Eukaryotic green algae such Chlamydomonas spp. have a similar but inducible $\mathrm{CCM}$ in which bicarbonate is concentrated in the chloroplast stroma via several $\mathrm{Ci}$ transporters and $\alpha$ and $\beta$ CAs. RubisCO is located in a specialized micro-compartment, the "pyrenoid”, where Ci fixation occurs [19].

An $\alpha \mathrm{CA}, \mathrm{CAH} 3$, is localized in the thylakoid lumen and is enriched in tubules that penetrate the pyrenoid. It plays an essential role in the rapid dehydration of the accumulated $\mathrm{HCO}_{3}{ }^{-}$that and thereby releases $\mathrm{CO}_{2}$ into the pyrenoid [20]. Mutants lacking CAH3 have a non-functional CCM in which $\mathrm{HCO}_{3}{ }^{-}$accumulates intracellularly. However, the mutant cells cannot grow at low levels of $\mathrm{CO}_{2}$ [21]. Furthermore, Limiting- $\mathrm{CO}_{2}$-inducible (LCI) $\mathrm{B}$ and $\mathrm{C}$ proteins are involved in $\mathrm{CO}_{2}$ metabolism. They form a complex of $350 \mathrm{kDa}$ localized around the pyrenoid in the light [22] and were initially thought to represent $\mathrm{Ci}$ transporters $[23,24]$. However, upon genetic analyses it was proposed that these proteins may trap $\mathrm{CO}_{2}$ released by $\mathrm{CAH} 3$ avoiding $\mathrm{CO}_{2}$ leakage [22,25]. These 
gene products are members of a small gene family so far only found in a few microalgae species [26]. Mutants with defects in B gene expression cannot grow in normal air but survive at very low $\mathrm{CO}_{2}$ indicating the existence of multiple $\mathrm{Ci}$ transporters in different $\mathrm{CO}_{2}$ conditions $[23,25,27]$. There is considerable evidence in green algae of a role in carbon capture of the plastid envelope via active transport of $\mathrm{Ci}$. Photosynthetically active chloroplasts as well as intact cells grown at high or low $\mathrm{CO}_{2}$ have low- or high-affinity $\mathrm{Ci}$ uptake systems, respectively [27].

Based on mathematical modeling of Chlamydomonas metabolism, it was proposed that an additional mitochondrial $\beta C A$ could be involved in converting $\mathrm{CO}_{2}$ from the TCA cycle or derived from photorespiration into $\mathrm{HCO}_{3}^{-}$which is transported via anaplerotic reactions (i.e. PEP carboxylase reaction to form 4 carbon acids) back into chloroplasts for carbon fixation by RubisCO, thereby potentially limiting $\mathrm{CO}_{2}$ leakage from mitochondria [28]. Such a role requires the presence of a bicarbonate translocase in the inner membrane of the mitochondrion, which has not yet been identified. This bicarbonate translocase would allow a controlled carbon efflux to the cytosol.

2.3 CCM in cyanobacteria The cyanobacterial CCM is based on a system that concentrates $\mathrm{HCO}_{3}{ }^{-}$ in the matrix using a series of carbonic anhydrases and $\mathrm{HCO}_{3}^{-}$transporters. Cyanobacterial RubisCO shows lower affinity for $\mathrm{CO}_{2}$ than that the enzyme present in of $\mathrm{C} 3$ plants. The higher $\mathrm{K}_{\mathrm{M}}$ of cyanobacterial RubisCO to $\mathrm{CO}_{2}$ is compensated by an efficient $\mathrm{CCM}$ that increases the $\left[\mathrm{CO}_{2}\right]$ concentration in around the carboxylating enzyme. Furthermore, RubisCO is localized within an icosahedral proteinaceous compartment called carboxysome ( $\alpha$ or $\beta$-type carboxysomes, see [29] for review depending on the species as reviewed in [29]). Mutants impaired in impairing functional CCM components, such as the carboxysome shell proteins or bicarbonate transporters, show very low photosynthetic affinity for external $\mathrm{Ci}$. They thus have a high $\mathrm{CO}_{2}$ requiring phenotype. These observations clearly demonstrate the importance of CCM for cyanobacterial survival in present atmospheric conditions. Both types of carboxysomes are composed of several shell proteins with 
different proposed functions. The main difference between $\alpha$ and $\beta$ carboxysomes is the RubisCO type (form A or B, respectively) and the operon encoding the shell proteins (the cso operon $c s o S 123 A B$ - or by the $c c m$ operon $-c c m K L M N$-, respectively) [30]. Bicarbonate dehydratation is catalyzed by a specific carboxysomal carbonic anhydrase that in the case of $\alpha$-carboxysome is an \&CA (encoded by csoS3 gene, [31]) and in $\beta$-carboxysome a $\beta \mathrm{CA}$, named CcaA, not integrated in the ccm operon [32]. One of the shell proteins of $\beta$-carboxysome, the CcmM subunit, consist of an its $\mathrm{N}$-terminus which resembles a gamma type carbonic anhydrases $(\gamma \mathrm{CAs})$ and a the $\mathrm{C}$-terminus which includes consists of RubisCO small subunit-like repeats [33]. CcmM is an important component subunit of a multiprotein bicarbonate dehydration complex which also includes, together with CcaA, and $\mathrm{CcmN}$ (similar to $\mathrm{CcmM}$ ). This complex, through $\mathrm{CcmM}$, also interacts with $\mathrm{CcmK}$ and $\mathrm{CcmL}$, the major shell proteins. $\mathrm{CcmM}$ is inactive as a carbonic anhydrase but has been proposed to be involved in the transport of bicarbonate to the inside of carboxysomes where it is converted by CcaA, into $\mathrm{CO}_{2}$ near RubisCO [34]. However, some $\beta$ cyanobacteria lack a functional ccaA gene. In these species, $\mathrm{CcmM}$ is an active carbonic anhydrase. The activity depends on an essential disulfide bond which is not conserved in other $\gamma \mathrm{CA}$ homologs [35]. Synechocystis spp. contain a third structurally related protein with a high similarity to bacterial and plant $\gamma \mathrm{CAs}$. However, its function or localization is unknown.

In addition, there is evidence for the association of CA-like proteins (Chp X and Y/Cup A and B) within to the NADH dehydrogenase complex (termed NDH-1 complex) of the thylakoid and plasma membranes of cyanobacteria. These polypeptides are involved in catalysing active $\mathrm{CO}_{2}$ uptake by converting $\mathrm{CO}_{2}$ into bicarbonate within the cell linked to electron transport and proton translocation associated with the NDH-1 complex. Although Chp/Cup proteins have no homologies with known CA protein families, two conserved histidine residues and one conserved cysteine residue which could act as a potential $\mathrm{Zn}$ coordination site have been identified (a novel CA class?). Electron donation to the complex by donors such as $\mathrm{NAD}(\mathrm{P}) \mathrm{H}$ produces a reduced intermediate within the NDH-1 complex that could oxidize the $\mathrm{Zn}-\mathrm{H}_{2} \mathrm{O}$ to $\mathrm{Zn}-\mathrm{OH}^{-}$and $\mathrm{H}^{+}$. In the second step, 
$\mathrm{Zn}-\mathrm{OH}$ could react with $\mathrm{CO}_{2}$ to form $\mathrm{HCO}_{3}$ which, and together with the abstracted released proton, is are translocated across the membrane to the lumen via a proton shuttle path within the hydrophobic proton channel subunits of the NDH-1 complex [36].

Because of the presence of efficient CCM systems, the finding of a high $\mathrm{CO}_{2}$ requiring phenotype, which completely lacks 2-phosphoglycolate metabolism (all three routes inactivated: oxalate decarboxylase -odc-, tartronic semialdehyde reductase $-t s r$ - and the plant-like glycine decarboxylase $-g c v T$ - or a double mutant in glycolate dehydrogenases $-g l c D 1$ and D2-) was unexpected and suggests that photosynthesis requires a functional CCM as well as a fully active glycolate detoxifying system [37]. By pulse labelling experiments using ${ }^{13} \mathrm{C} \mathrm{NaHCO}_{3},{ }^{13} \mathrm{C}$ glycolate was detected under conditions thought to suppress photorespiration [38]. Therefore, both CCM and 2-phosphoglycolate metabolism appear to be crucial for the viability of all organisms performing oxygenic photosynthesis (cyanobacteria and plants), grown at normal $\mathrm{CO}_{2}$ conditions.

\section{Proposed basal CCM}

The discovery of $\gamma \mathrm{CAs}$ in mitochondria of almost all photosynthetic eukaryotic organisms analyzed so far but not in animals or fungi led to hypothesize on their physiological role. The mitochondrial $\gamma \mathrm{CAs}$ are attached to complex I (CI) of the respiratory chain and form a spherical extra domain (named CA domain) on the matrix exposed side of its membrane arm [39].

A possible hypothesis extends a proposition originally made by Prof. John A. Raven [28] that bicarbonate translocases possibly present in the inner mitochondrial membrane in Chlamydomonas spp. are involved in recapturing $\mathrm{Ci}$ from mitochondrial decarboxylations reactions. We propose that the CA domain of complex I forms part of a mitochondrial bicarbonate export system that allows efficient transfer $\mathrm{Ci}$ from mitochondria to chloroplasts. The $\mathrm{CA}$ domain is 
involved in bicarbonate formation and/or bicarbonate transfer across the inner mitochondrial membrane. Additionally, the CA domain could produce and translocate bicarbonate. Especially in the presence of high light or temperature, $\mathrm{CO}_{2}$ in chloroplasts is usually low due to ribulose-1,5bisphosphate carboxylation by RubisCO and due to the fact that stomata can be closed at daytime and consequently import of atmospheric $\mathrm{CO}_{2}$ into the leaf is restricted. Chloroplast $\mathrm{CO}_{2}$ levels are usually low due to ribulose-1,5-bisphosphate carboxylation by RubisCO and due to . At the same time, import of atmospheric $\mathrm{CO} 2$ into the leaf often is restricted because the stomata are closed. and when stomata are closed, especially in the presence of high light or temperature, restricting import of atmospheric $\mathrm{CO}_{2}$ into the leaf. At the same time, mitochondria produce excess $\mathrm{CO}_{2}$ due to the decarboxylation reactions of the TCA cycle but especially due to glycine - serine conversion from photorespiration. While $\mathrm{CO}_{2}$ is more or less "waste" in animal and fungal mitochondria, in plant cells it is the main substrate for photosynthetic carbon fixation in chloroplasts and, at many conditions, rate-limiting for plant growth. Part of the mitochondrial $\mathrm{CO}_{2}$ can be re-cycled for carbon fixation by RubisCO following diffusion to the chloroplast. However, we additionally propose occurrence of a more targeted carbon transfer system which, besides other elements, is constituted by a bicarbonate export system linked to mitochondrial complex I. Bicarbonate could be transferred from the cytosol into chloroplasts by another bicarbonate translocase or by a metabolic system resembling the carbon pre-fixation steps during $\mathrm{C} 4$-metabolism. In the chloroplasts, $\beta \mathrm{CAs}$ facilitate efficient re-conversion of bicarbonate into $\mathrm{CO}_{2}$ for carbon fixation by RubisCO. Our hypothesis is summarized in Figure 1. Furthermore, we propose that the CA domain of complex I has a function functions like the carboxysomal CcmM-CcmN proteins. Plant proteins could have evolved from $\gamma \mathrm{CAs}$ of an ancient $\alpha$ protobacterium during evolution of the mitochondrial compartment. the adaptation of the first endosymbiont to be converted into a mitochondrion. These proteins are were only conserved in the plant and basal lineages of Eukarya. This could be regarded as a case of convergent evolution. Thus, We propose that all photosynthetic eukaryotic organisms contain this "basal" CCM (bCCM, Figure 1) and that this process could account for about 10 to $20 \%$ of carbon 
fixation in $\mathrm{C} 3$ plants. In the following sections, we present recent experimental results which, according to our interpretation, support the hypothesized basal carbon transfer mechanism within plant cells.

\subsection{Evidence for the basal CCM}

Mitochondrial $\gamma \mathrm{CAs}$ have been described for Arabidopsis (C3, dicotyledonous), Rice (C3, monocotyledonous), Maize (C4 monocotyledonous) as well as in for some green algae such as Chlamydomonas (C3 with CCM), [39-46]. Surprisingly, no $\gamma \mathrm{CA}$ homologs were found in the Ostreococcus tauri genome, which is the smallest known eukaryotic green alga [47]. Recently, similar proteins have been found in mitochondrial complex I of Acanthomoeba castellani and other aplastidic eukaryotes such as Amoebazoa, Chromalveolata and Excavata. [48,49]. It can be assumed that the function of $\gamma \mathrm{CA}$ proteins is somewhat different in plant/green algae than in aplastidic eukaryotes.

In Arabidopsis, the gammaCA family consists of five proteins encoded by five nuclear genes. Three are named $\gamma \mathrm{CA} 1, \gamma \mathrm{CA} 2$ and $\gamma \mathrm{CA} 3$ because they very much resemble the "prototype" $\gamma \mathrm{CA}$ from Metanosarcina thermophila (Cam) and two are named $\gamma \mathrm{CAL} 1$ and $\gamma \mathrm{CAL} 2$ ("gamma Carbonic Anhydrase like") because they have a more derived sequence with respect to Cam with less conservation of important residues [50]. At the same time, the Arabidopsis CA/CAL proteins exhibit sequence similarity to cyanobacterial $\mathrm{CcmM}$ proteins. The $\mathrm{CA} / \mathrm{CAL}$ proteins form the so called "CA domain" of complex I first discovered in Arabidopsis [44, 39]. The exact composition of this domain is not known in detail. Based on single particle electron microscopy, the CA domain most likely is a trimer [39,45,51], which nicely corresponds to the composition of Cam [52]. Since the interaction between $\gamma$ CALs and $\gamma$ CAs is strong and since all photosynthetic organisms analyzed so far contain at least one $\gamma \mathrm{CA}$ and one $\gamma \mathrm{CAL}$, the CA domain most likely represents a heterotrimer 
[50]. It so far is unclear if all five CA/CAL proteins of Arabidopsis are simultaneously present in individual complex I particles. All five proteins were detected by mass spectrometry within isolated complex I or within the $550 \mathrm{kDa}$ membrane arm of this complex upon dissection of the holoenzyme by low SDS $(0,01 \%)$ [46]. Moreover, at slightly higher SDS concentrations $(0.04 \%)$, a $~ 85$ $\mathrm{kDa}$ subcomplex is detached from Complex I which includes $\gamma \mathrm{CA} 1$ and $\gamma \mathrm{CA} 2$ and the two $\gamma$ CALs but surprisingly not $\gamma \mathrm{CA} 3$. Additional data suggest that $\gamma \mathrm{CAL} 1$ and $\gamma \mathrm{CAL} 2$ are not simultaneously present within individual CA subcomplexes [46]. Therefore, the CA domain of complex I seems to be composed by three CA/CAL proteins of varying identity. The physiological relevance of this heterogeneity is currently a mystery.

It so far has not been possible to measure carbonic anhydrase activity of the mitochondrial $\gamma \mathrm{CAs}$ for any plant or eukaryotic green algae although all relevant amino acids are present and properly arranged in a putative active site similar to Cam or members of a Cam subfamily termed $\mathrm{CamH}$ [42]. However, it was shown that cyanobacterial CcmM as well as Arabidopsis $\gamma \mathrm{CA} 2$ bind $\mathrm{Ci}$ at comparable rates [34, 53]. Amino acids essential to bind bicarbonate are also conserved [42]. Thus we propose that the CA domain of complex I could function as a carbonic anhydrase and/or bicarbonate translocator. Bicarbonate could bind the conserved GIn and Tyr residues together with a possible metal ion $\left(\mathrm{Zn}^{2+}\right.$ or $\left.\mathrm{Fe}^{2+}\right)$. How the bicarbonate could be transferred across the mitochondrial membrane? This currently is completely speculative. Interestingly, EM analysis of complex I from Arabidopsis revealed presence of a small cavity within its membrane arm in opposite to the point of attachment of the CA domain, possibly indicating a pore like structure and this position [44]. Furthermore, protease protection experiments indicate that the CA subunits of Arabidopsis span the membrane once and that their C-termini constitute a small domain on the intermembrane-space exposed side of the membrane arm [39]. In the micrographs of several plantlike complexes I, a cavity is clearly seen opposed to the CA domain [44] and for some experimental observations [39], the C-terminus of CA proteins would transverse the membrane exposing $2 \mathrm{kDa}$ to 
the intermembrane space. The C-termini of more than one subunit These C-termini could form an amphipathic channel which allows bicarbonate transfer, possibly together with protons where bicarbonate could pass possibly helped by proton transfer that could be associated as it was proposed for Cup proteins in the NDH-1 complexes in cyanobacteria [36].

Gene expression profiling data indicate that mitochondrial $\gamma$ CAs are down-regulated when Arabidopsis is cultivated at elevated $\mathrm{CO}_{2}$ concentrations [54]. These data indicate a possible role of the CA proteins during photorespiration [51]. Furthermore, ethoxzolamide (EZA), a strong inhibitor of $\gamma$ CAs (and to a lesser extent of $\beta C A s$ ) impairs photosynthetic oxygen evolution in isolated protoplasts but not in isolated chloroplasts. This effect was only observed at low $\mathrm{CO}_{2}$ concentrations with almost no effect at high $\mathrm{CO}_{2}$ concentrations [55]. These results strongly suggest that internal CAs (outside the chloroplast) are involved in recycling of mitochondrial $\mathrm{CO}_{2}$ in photorespiratory conditions.

Arabidopsis null mutant plants lacking the gene encoding $\gamma \mathrm{CA} 2$ contain drastically reduced amounts of complex I. A weaker effect was found in ca3 null mutant plants. Moreover, oxygen consumption experiments carried out in the presence of different respiratory chain inhibitors using leaves and flower tissues as well as cell suspensions growing in the dark indicate that these mutant plants have increased alternative respiration. Both mutant lines did not exhibit an altered phenotype with respect of wild type plants at conditions so far tested but derived cell suspensions are growing more slowly than wild type in darkness [54].

Arabidopsis plants overexpressing $\gamma \mathrm{CA} 2$ plants show a male sterile phenotype by indehiscence of anthers due to a dramatic reduction in ROS content which seems to cause reduced lignin deposition [56] Furthermore, these plants have significantly longer roots and bigger seeds. A considerable enrichment of $\gamma \mathrm{CA} 2$ within Complex I was consistently detected (Villarreal and 
Zabaleta, unpublished results) suggesting that over-expression of this protein leads to replacement of $\gamma \mathrm{CA} 1$ and $\gamma \mathrm{CA} 3$ within the trimeric CA domain attached to complex I. Homotrimers of $\gamma \mathrm{CA} 2$ bind $\mathrm{Ci}$ as efficiently as the cyanobacterial $\mathrm{CcmM}$ protein [34,53]. Both proteins are assumed to have a similar function in $\mathrm{Ci}$ translocation, which is especially important in the context of photorespiration [51].

Complex I abundance and activity is normal in mitochondria of plants overexpressing $\gamma \mathrm{CA} 2$. If the CA domain of complex I indeed is involved in mitochondrial bicarbonate export, a possible explanation of the observed bigger sink organs is that increased abundance of $\gamma \mathrm{CA} 2$ should allow higher rates of bicarbonate export resulting in higher $\mathrm{CO}_{2}$ fixation rates by RubisCO and thus increasing plant growth. This increase in plant growth only can take place if $\gamma \mathrm{CA} 2$ over-expression does not impair respiration. Indeed, oxygen consumption experiments using an Arabidopsis cell suspension culture overexpressing $\gamma \mathrm{CA} 2$ revealed elevated oxygen consumption rates and faster growth (approx. $70 \%$ more fresh weight per week compared to wild type cells, Villarreal and Zabaleta, unpublished results).

It so far is unknown how bicarbonate gets into the chloroplasts since a cyanobacterial-like bicarbonate transporter or equivalent has not yet been identified in higher plants. One possibility is that bicarbonate exported from mitochondria reacts with PEP. The reaction is catalysed by a cytoplasmic PEP carboxylase. The resulting oxaloacetate can be converted into malate and transported into chloroplasts as in C4 metabolism (Figure 1). All necessary enzymes are present in Arabidopsis (cytosolic AtPEP carboxylase [57], cytosolic malate dehydrogenase, E.C. 1.1.1.83 antiporter for malate / oxaloacetate exchange, dicarboxylic acid translocase AtDiT family, [58] and a chloroplast NADP malic enzyme, E.C. 1.1.1.40, [59,60]). The Arabidopsis dicarboxylic acid translocase DiT2 knockout mutant, dct2 and tobacco DiT1 antisense plants both have a photorespiratory phenotype $[57,61]$. It has also been shown that DiT1 has a high affinity for the 
dicarboxylate oxaloacetate [61]. These evidences are consistent with the interpretation that malate or oxaloacetate transporters are important during photorespiration, according to our hypothesis for recycling mitochondrial $\mathrm{Ci}$ to carbon fixation by RubisCO. The net result is the introduction of $\mathrm{CO}_{2}$ into the chloroplast. However, recent comparative proteome and transcriptomic analyses with C3 and C4 plants revealed that the DiT proteins specifically are enriched in the chloroplast envelopes of C4 plants [62, 63]. The DiT family members may play a role in central nitrogen metabolism [61] and for core C4 photosynthesis in maize [64]. All members of the DiT families were identified in $\mathrm{C} 4$ as well as in $\mathrm{C} 3$ plants. A recent investigation clearly showed that all enzymes required for C4 photosynthesis are present in Arabidopsis and that cell specific expression of the corresponding transcripts in C4 species can be explained by as yet unidentified trans-factor [65]. Several reports indicate as well that high activities of the C4 enzymes are found around the veins in $\mathrm{C} 3$ species and may have facilitated the polyphyletic evolution of $\mathrm{C} 4$ photosynthesis [66].

CMS I and II Nicotiana sylvestris cytoplasmic male sterile mutants have drastically reduced amounts of complex I, which affects photosynthesis under normal (photorespiratory) conditions [67]. Steady-state photosynthesis in the mutant was reduced by $20 \%$ to $30 \%$ at atmospheric $\mathrm{CO}_{2}$ levels. The inhibition of photosynthesis was alleviated by high $\mathrm{CO}_{2}$ or low $\mathrm{O}_{2}$. It was interpreted that a functional complex I is required to ensure a subcellular redox balance $[3,68]$. However, the importance of complex I for photosynthesis also can be explained by its proposed role in the recycling of mitochondrial $\mathrm{Ci}$.

Taken together, the proposed basal CCM for efficient recycling of mitochondrial $\mathrm{CO}_{2}$ for carbon fixation in chloroplasts, which is especially important during photorespiratory conditions, is supported by several experimental findings: (i) the CA domain of complex I is present in all plants but absent in animal and fungal mitochondria; (ii) CA proteins efficiently can bind $\mathrm{Ci}$ and very much resemble the cyanobacterial $\mathrm{CcmM}$ proteins involved in the enrichment of $\mathrm{Ci}$ in 
carboxysomes; (iii) genes encoding CA proteins are down-regulated when plants are cultivated at elevated $\mathrm{CO}_{2}$; (iv) mutants with reduced amounts of complex I have reduced photosynthesis rates under photorespiratory but not under non-photorespiratory conditions and (v) other components of the proposed $\mathrm{Ci}$ transfer system, which are necessary for $\mathrm{Ci}$ transfer into chloroplasts, might represent enzymes of the carbon pre-fixation step of $\mathrm{C} 4$ metabolism. All necessary enzymes are also present in C3 plants. Interestingly, some mutants of these enzymes have photorespiratory phenotypes.

\section{Outlook}

Photorespiration is a metabolic process of great importance. It is one of the main determining factors for biomass production in C3 crops. Recent technological developments have allowed to address important questions concerning the photorespiratory pathway (reviewed in [3]). However, a number of aspects deserve further investigation, especially the role of metabolite transporters such as the dicarboxylic acid translocases in photorespiration and the role of the photorespiratory enzymes such as PEP carboxylase and NADP malic enzyme in C3 plants. Last but not least, the physiological role of the CA domain of complex I, which not only occurs in C3 plants but also in plants with other photosynthetic metabolism and in green algae, has to be further investigated. Specifically, bicarbonate export by complex I has to be analyzed, e.g. by reconstitution of plant complex I into artificial liposomes.

The proposed basal CCM involves a number of metabolites which are also part the photorespiratory [69] and other cellular pathways. Its investigation therefore is a complicated task. RNA profiling and proteomic analyses of isolated organelles [62, 70-72] may help understand this network. Isolation of organelles from different tissues or cell types will also provide interesting data about the function of the proposed basal CCM. For example, the CA domain of complex I is present 
in C4 maize [45]. Do mitochondria of mesophyll as well as bundle sheath cells contain the same arrangement of complex I subunits? Future research should also investigate other organisms with differences respect to photosynthetic primary metabolism, for example green algae. The CA domain is also present in these organisms, which at the same time contain other sophisticated CCM systems. All these investigations should be of fundamental importance to achieve a better understanding of the evolution of cyanobacterial CCM to the compartmentalized (yes, this term exists and is correct in this context!!) basal CCM proposed for plants.

In the last decade, several projects have attempted to improve the photosynthetic performance of $\mathrm{C} 3$ crop plants. One idea is to incorporate $\mathrm{C} 4 \mathrm{CCM}$ into $\mathrm{C} 3$ crop (mainly rice) and thereby elevating $\mathrm{CO}_{2}$ around RubisCO [73-76]. A second approach is to transfer cyanobacterial bicarbonate transporters or even other cyanobacterial $\mathrm{CCM}$ proteins to $\mathrm{C} 3$ chloroplasts to provide significant improvement in photosynthetic performance [77]. A third approach is based on the idea that - since the oxygenation reaction of RubisCO can not be eliminated - the degradation of 2phosphoglycolate could be improved via a bacterial glycolate pathway introduced in Arabidopsis chloroplasts [78]. In a fourth approach, the complete design of a novel pathway to fully oxidizes 2phosphoglycolate to $\mathrm{CO}_{2}$ was undertaken based on the incorporation of glycolate oxidase, malate synthase and catalase into Arabidopsis chloroplasts [79]. And finally: alterations of the CA domain of complex I might allow to optimize photosynthesis in crops. The combination of more than one approach might one day allow developing higher yielding crops for feeding tomorrow's world.

\section{Acknowledgements}

This research was supported by a bi-national program for the project related exchange of scientists between Argentina and Germany financed by the Ministerio de Ciencia, Tecnología e Innovación 
Productiva (MINCyT) and the Deutsche Akademische Austauschbung (DAAD). Furthermore, research of HPB is supported by the Deutsche Forschungsgemeinschaft (Grant Br 1829/10-1) and of EZ by the Agencia Nacional de Promoción Científica y Tecnológica, Argentina (ANPCyT 01673). 


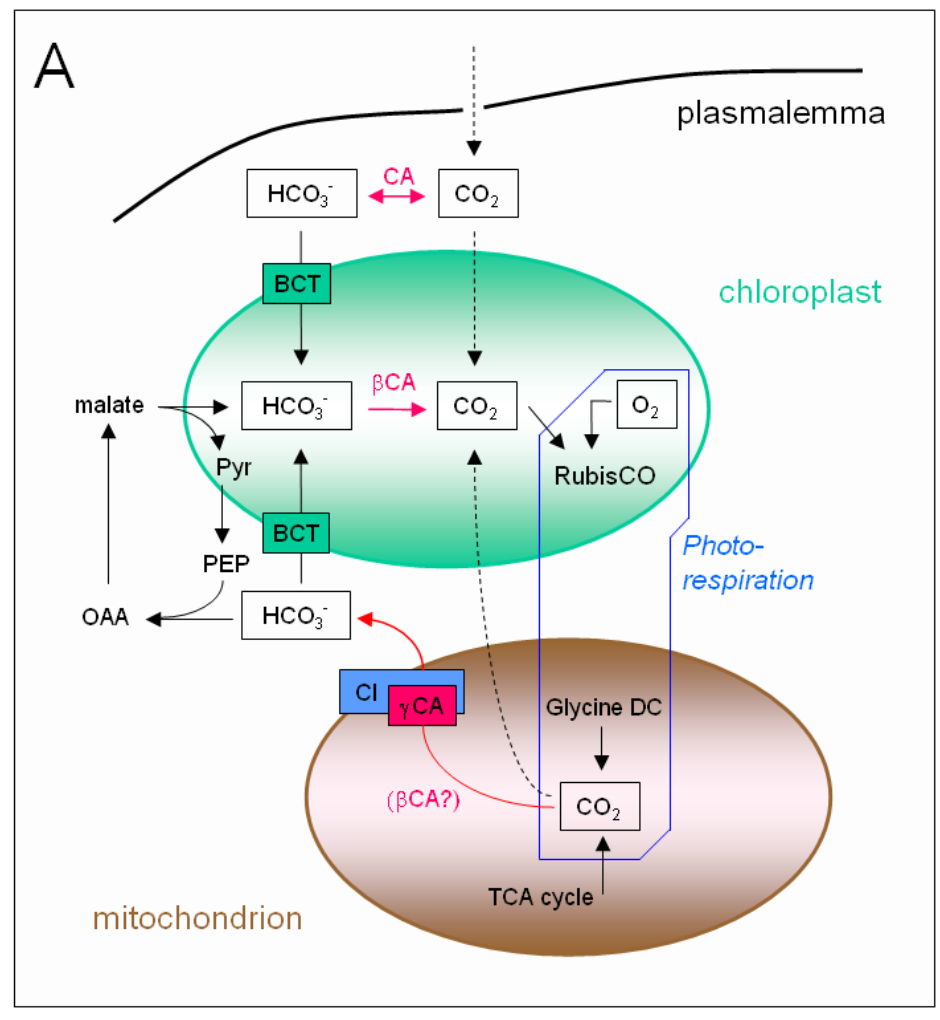

Basal CCM of plant cells

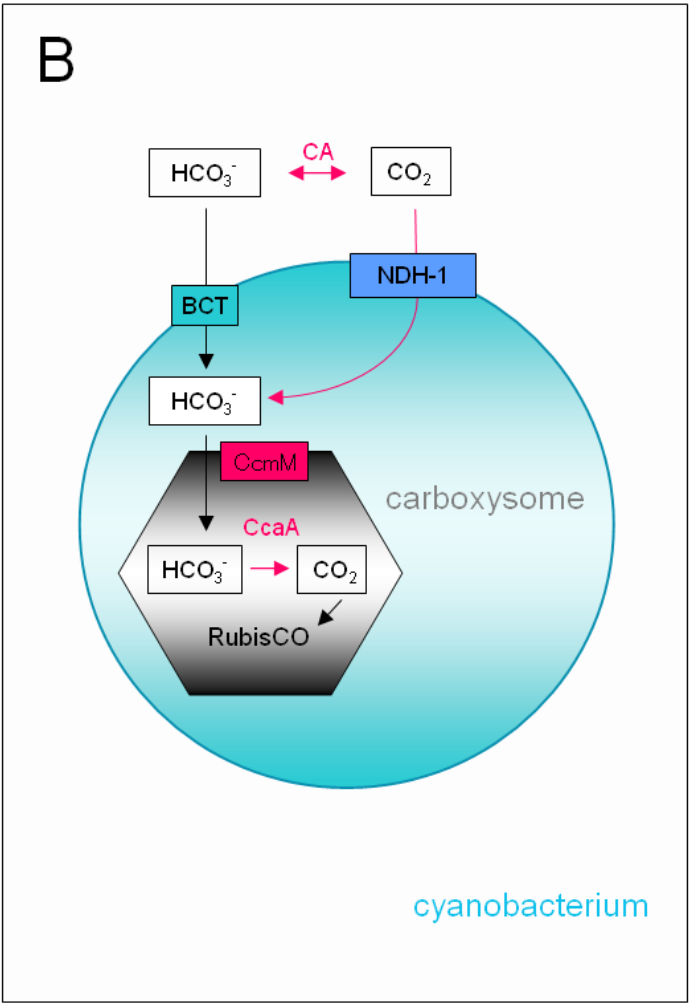

CCM of cyanobacteria

\section{Figure 1:}

\section{Model for Carbon Concentrating Mechanism in Plants and Cyanobacteria}

A- Proposed basal CCM (bCCM) in plants. The $\mathrm{CO}_{2}$ concentration in chloroplasts often is low due to carbon fixation by RubisCO, especially if plants are cultivated under high light or at high temperatures. At the same time, large amounts of $\mathrm{CO}_{2}$ are liberated in mitochondria due to the reactions of the TCA cycle and photorespiration. Experimental evidence indicates that mitochondrial $\mathrm{CO}_{2}$ is recycled for carbon fixation in chloroplasts. Transfer of $\mathrm{CO}_{2}$ could be based on diffusion but would be more efficient with an active $\mathrm{HCO}_{3}{ }^{-}$ transfer mechanism. The mitochondrial CA domain (given in red) of complex I (blue) is proposed to play a role in converting $\mathrm{CO}_{2}$ to $\mathrm{HCO}_{3}{ }^{-}$and/or transfer $\mathrm{HCO}_{3}{ }^{-}$from mitochondria into the cytosol. A matrixlocalized $\beta C A$ (At1g58180) might additionally be involved in $\mathrm{HCO}_{3}{ }^{-}$formation or only transfer if conversion is catalysed by a mitochondrial $\beta C A$ (At1g58180). Subsequently, $\mathrm{HCO}_{3}{ }^{-}$could be transferred from the cytosol into the chloroplast by a putative bicarbonate translocator in the chloroplast envelope or by a C4-like pathway. B- Carbon concentration mechanism (CCM) of cyanobacteria. Cyanobacteria export a carbonic anhydrase increasing the bicarbonate concentration in their surroundings. Transporters are localized in the plasma membrane of cyanobacteria facilitating active uptake of bicarbonate or $\mathrm{CO}_{2}$. Besides other components, cyanobacterial complex I (NDH-1; given in blue) is involved in this process. Bicarbonate subsequently is transferred into a specialized compartment, the "carboxysome", where $\mathrm{CO}_{2}$ fixation occurs. Capturing of inorganic carbon within the carboxysome Translocation is based on the CcmM protein (given in red) and conversion of bicarbonate into $\mathrm{CO}_{2}$ by on CcaA. Designations: $\mathrm{CI}$ : mitochondrial complex I, NDH1: cyanobacterial complex I involved in $\mathrm{Ci}$ uptake, BCT: bicarbonate translocase, $\beta \mathrm{CA}$ and $\gamma \mathrm{CA}$ : beta- and gamma-type carbonic anhydrases, $\mathrm{CcmM}$ and $\mathrm{CcaA}$ : carboxisomal proteins involved in $\mathrm{Ci}$ delivery for RubisCO, OAA: oxaloacetate, Pyr: pyruvate, PEP: phosphoenolpyruvate. 


\section{References}

[1] R.F. Sage, P.A. Christin, E.J. Edwards, The C4 plant lineages of planet Earth, J. Exp. Bot. 62 (2011) 3155-3169.

[2] S. Timm, A. Florian, K. Jahnke, A. Nunes-Nesi, A.R. Fernie, H. Bauwe, The hydroxypyruvate-reducing system in Arabidopsis: multiple enzymes for the same end, Plant Physiol. 155 (2011) 694-705.

[3] H. Bauwe, M. Hagemann, A.R. Fernie, Photorespiration: players, partners and origin, Trends Plant Sci. 15 (2010) 330-336.

[4] S.K. Klavsen, T.V. Madsen, S.C. Maberly, Crassulacean acid metabolism in the context of other carbonconcentrating mechanisms in freshwater plants: a review, Photosynth Res. 109 (2011) 269-279.

[5] M. Ludwig, The molecular evolution of $\beta$-carbonic anhydrase in Flaveria, J. of Exp. Bot., 62 (2011) 3071-3081.

[6] R.F. Sage, X.G. Zhu, Exploiting the engine of C4 photosynthesis, J Exp Bot. 62 (2011) 2989-3000.

[7] J.B. Reiskind, G. Bowes, The role of phosphoenol pyruvate carboxykinase in a marine macroalga with C4-like photosynthetic characteristics, Proc. Natl. Acad. Sci. USA 88 (1991) 2883-2887.

[8] J.R. Reinfelder, A.M. Kraepiel, F.M. Morel, Unicellular C4 photosynthesis in a marine diatom, Nature 407 (2000) 996-999.

[9] J.R. Reinfelder, A.J. Milligan, F.M. Morel, The role of the C4 pathway in carbon accumulation and fixation in a marine diatom, Plant Physiol. 135 (2004) 2106-2111.

[10] J.A. Raven, Inorganic carbon acquisition by eukaryotic algae: four current questions, Photosynth Res. 106 (2010) $123-134$.

[11] P.J. Vogan, M.W. Frohlich, R.F. Sage, The functional significance of C3-C4 intermediate traits in Heliotropium L. (Boraginaceae): gas exchange perspectives, Plant Cell Environ. 30 (2007) 1337-1345.

[12] A.D. McKown, J.M. Moncalvo, N.G. Dengler, Phylogeny of Flaveria (Asteraceae) and inference of C4 photosynthesis evolution, Am. J. Bot. 92 (2005) 1911-1928.

[13] O. Ueno, Environmental regulation of $\mathrm{C} 3$ and $\mathrm{C} 4$ differentiation in the amphibious sedge Eleocharis vivipara, Plant Physiol. 127 (2001) 1524-1532.

[14] E.V. Voznesenskaya, V.R. Franceschi, O. Kiirats, H. Freitag, G.E. Edwards, Kranz anatomy is not essential for terrestrial C4 plant photosynthesis, Nature 414 (2001) 543-546.

[15] S.D. Chuong, V.R. Franceschi, G.E. Edwards, The cytoskeleton maintains organelle partitioning required for single-cell C4 photosynthesis in Chenopodiaceae species, Plant Cell 18 (2006) 2207-2223.

[16] S. Offermann, T.W. Okita, G.E. Edwards, Resolving the compartmentation and function of C4 photosynthesis in the single-cell C4 species Bienertia sinuspersici, Plant Physiol. 155 (2011) 1612-1628.

[17] I. Zelitch, N.P. Schultes, R.B. Peterson, P. Brown, T.P. Brutnell, High glycolate oxidase activity is required for survival of maize in normal air, Plant Physiol. 149 (2009) 195-204.

[18] V.G. Maurino, C. Peterhänsel, Photorespiration: current status and approaches for metabolic engineering, Curr Opin. Plant Biol. 13 (2010) 249-256.

[19] J.V. Moroney, R.A. Ynalvez, Proposed carbon dioxide concentrating mechanism in Chlamydomonas reinhardtii, Eukaryot Cell. 6 (2007) 1251-1259.

[20] M. Mitra, S.M. Lato, R.A. Ynalvez, Y. Xiao, J.V. Moroney, Identification of a new chloroplast carbonic anhydrase in Chlamydomonas reinhardtii. Plant Physiol. 135 (2004) 173-182. 
[21] J. Karlsson, A.K. Clarke, Z.Y. Chen, S.Y. Hugghins, Y.I. Park, H.D. Husic, J.V. Moroney, G. Samuelsson, A novel alpha-type carbonic anhydrase associated with the thylakoid membrane in Chlamydomonas reinhardtii is required for growth at ambient $\mathrm{CO}_{2}$, EMBO J. 17 (1998) 1208-1216.

[22] T. Yamano, T. Tsujikawa, K. Hatano, S. Ozawa, Y. Takahashi, H. Fukuzawa, Light and low-CO ${ }_{2}$-dependent LCIBLCIC complex localization in the chloroplast supports the carbon-concentrating mechanism in Chlamydomonas reinhardtii, Plant Cell Physiol. 51 (2010) 1453-1468.

[23] Y. Wang, M.H. Spalding, An inorganic carbon transport system responsible for acclimation specific to air levels of $\mathrm{CO}_{2}$ in Chlamydomonas reinhardtii, Proc Natl Acad Sci USA. 103 (2006) 10110-10115.

[24] Y. Wang, D. Duanmu, M.H. Spalding, Carbon dioxide concentrating mechanism in Chlamydomonas reinhardtii: inorganic carbon transport and $\mathrm{CO}_{2}$ recapture, Photosynth Res. 109 (2011) 115-122.

[25] D. Duanmu, Y. Wang, M.H. Spalding, Thylakoid lumen carbonic anhydrase (CAH3) mutation suppresses air-Dier phenotype of LCIB mutant in Chlamydomonas reinhardtii, Plant Physiol. 149 (2009) 929-937.

[26] M.H. Spalding, Microalgal carbon-dioxide-concentrating mechanisms: Chlamydomonas inorganic carbon transporters., J. Exp. Bot. 59 (2008) 1463-1473.

[27] D. Duanmu, A.R. Miller, K.M. Horken, D.P. Weeks, M.H. Spalding, Knockdown of limiting-CO ${ }_{2}$-induced gene HLA3 decreases $\mathrm{HCO}_{3}{ }^{-}$transport and photosynthetic Ci affinity in Chlamydomonas reinhardtii, Proc Natl Acad Sci USA. 106 (2009) 5990-5995.

[28] J.A. Raven, A role for mitochondrial carbonic anhydrase in limiting $\mathrm{CO}_{2}$ leakage from low $\mathrm{CO}_{2}$-grown cells of Chlamydomonas reinhardtii, Plant, Cell Environ. 24 (2001) 261-265.

[29] G.D. Price, Inorganic carbon transporters of the cyanobacterial $\mathrm{CO}_{2}$ concentrating mechanism, Photosynth Res. 109 (2011) 33-45

[30] M.R. Badger, G.D. Price, $\mathrm{CO}_{2}$ concentrating mechanisms in cyanobacteria: molecular components, their diversity and evolution, J. Exp. Bot. 54 (2003) 609-622.

[31] A.K. So, G.S. Espie, E.B. Williams, J.M. Shively, S. Heinhorst, G.C. Cannon, A novel evolutionary lineage of carbonic anhydrase (epsilon class) is a component of the carboxysome shell, J. Bacteriol. 186 (2004) 623-630.

[32] A.K. So, G.S. Espie, Cloning, characterization and expression of carbonic anhydrase from the cyanobacterium Synechocystis PCC6803, Plant Mol. Biol. 37 (1998) 205-215.

[33] M. Ludwig, D. Sültemeyer, G.D. Price, Isolation of ccmKLMN genes from the marine cyanobacterium Synechococcus sp. PCC7002 (Cyanophyceae) and evidence that CcmM is essential for carboxysome assembly, J. Phycol. 36 (2000) 1109-1118.

[34] S.S. Cot, A.K. So, G.S. Espie, A multiprotein bicarbonate dehydration complex essential to carboxysome function in cyanobacteria, J. Bacteriol. 190 (2008) 936-945.

[35] K.L. Peña, S.E. Castel, C. de Araujo, G.S. Espie, M.S. Kimber, Structural basis of the oxidative activation of the carboxysomal gamma-carbonic anhydrase, CcmM, Proc. Natl. Acad. Sci. USA. 107 (2010) 2455-2460.

[36] S. Maeda, M.R. Badger, G.D. Price, Novel gene products associated with NdhD3/D4-containing NDH-1 complexes are involved in photosynthetic $\mathrm{CO}_{2}$ hydration in the cyanobacterium, Synechococcus sp. PCC7942, Mol Microbiol. 43 (2002) 425-435.

[37] M. Eisenhut, W. Ruth, M. Haimovich, H. Bauwe, A. Kaplan, M. Hagemann, The photorespiratory glycolate metabolism is essential for cyanobacteria and might have been conveyed endosymbiontically to plants, Proc. Natl. Acad. Sci. USA. 105 (2008) 17199-17204.

[38] J. Huege, J. Goetze, D. Schwarz, H. Bauwe, M. Hagemann, J. Kopka, Modulation of the Major Paths of Carbon in Photorespiratory Mutants of Synechocystis, PLoS ONE 6 (2011) e16278. doi:10.1371/journal.pone.0016278. 
[39] S. Sunderhaus, N.V. Dudkina, L. Jänsch, J. Klodmann, J. Heinemeyer, M. Perales, E. Zabaleta, E.J. Boekema, , HP. Braun, Carbonic anhydrase subunits form a matrix-exposed domain attached to the membrane arm of mitochondrial complex I in plants, J Biol Chem. 281 (2006) 6482-6488.

[40] V. Kruft, H. Eubel, L. Jänsch, W. Werhahn, H-P. Braun, Proteomic approach to identify novel mitochondrial proteins in Arabidopsis, Plant Physiol. 127 (2001) 1694-1710.

[41] J.L. Heazlewood, K.A. Howell, A.H. Millar, Mitochondrial complex I from Arabidopsis and rice: orthologs of mammalian and fungal components coupled with plant-specific subunits, Biochim Biophys Acta. 1604 (2003) 159-169.

[42] G. Parisi, M. Perales, M.S. Fornasari, A. Colaneri, N. González-Schain, D. Gómez-Casati, S. Zimmermann, A. Brennicke, A. Araya, J.G. Ferry, J. Echave, E. Zabaleta, Gamma carbonic anhydrases in plant mitochondria, Plant Mol. Biol. 55 (2004) 193-207.

[43] P. Cardol, F. Vanrobaeys, B. Devreese, J. Van Beeumen, R.F. Matagne, C. Remacle, Higher plant-like subunit composition of mitochondrial complex I from Chlamydomonas reinhardtii: 31 conserved components among eukaryotes, Biochim Biophys Acta. 1658 (2004) 212-224.

[44] N.V. Dudkina, H. Eubel, W. Keegstra, E.J. Boekema, H-P. Braun, Structure of a mitochondrial supercomplex formed by respiratory-chain complexes I and III, Proc. Natl. Acad. Sci. U S A. 102 (2005) 3225-3229.

[45] K. Peters., N.V. Dudkina, L. Jänsch, H-P. Braun, E.J. Boekema, A structural investigation of complex I and I+III2 supercomplex from Zea mays at 11-13 A resolution: assignment of the carbonic anhydrase domain and evidence for structural heterogeneity within complex I, Biochim Biophys Acta. 1777 (2008) 84-93.

[46] J. Klodmann, S. Sunderhaus, M. Nimtz, L. Jänsch, H-P. Braun, Internal architecture of mitochondrial complex I from Arabidopsis thaliana, Plant Cell 22 (2010) 797-810.

[47] E. Derelle, C. Ferraz, S. Rombauts, et al. Genome analysis of the smallest free-living eukaryote Ostreococcus tauri unveils many unique features, Proc. Natl. Acad. Sci. U S A. 103 (2006) 11647-11652.

[48] R.M. Gawryluk, M.W. Gray, Evidence for an early evolutionary emergence of gamma-type carbonic anhydrases as components of mitochondrial respiratory complex I, BMC Evol. Biol. 10 (2010) 176-179.

[49] P. Cardol, Mitochondrial NADH:ubiquinone oxidoreductase (complex I) in eukaryotes: A highly conserved subunit composition highlighted by mining of protein databases, Biochim. Biophys Acta. 1807 (2011) 1390-1397.

[50] M. Perales, G. Parisi, M.S. Fornasari, A. Colaneri, F. Villarreal, N. González-Schain, J. Echave, D. Gómez-Casati, H-P. Braun, A. Araya, E. Zabaleta, Gamma carbonic anhydrase like complex interact with plant mitochondrial complex I, Plant Mol. Biol. 56 (2004) 947-957.

[51] H-P. Braun, E. Zabaleta, Carbonic anhydrase subunits of the mitochondrial NADH dehydrogenase complex (complex I) in plants, Physiol. Plant. 129 (2007) 114-122.

[52] B.E. Alber, J.G. Ferry, Characterization of heterologously produced carbonic anhydrase from Methanosarcina thermophila. J. Bacteriol. 178 (1996) 3270-3274.

[53] V. Martin, F. Villarreal, I. Miras, A. Navaza, A. Haouz, R.M. González-Lebrero, S.B. Kaufman, E. Zabaleta, Recombinant plant gamma carbonic anhydrase homotrimers bind inorganic carbon, FEBS Lett. 583 (2009) 3425-3430.

[54] M. Perales, H. Eubel, J. Heinemeyer, A. Colaneri, E. Zabaleta, H-P. Braun Disruption of a nuclear gene encoding a mitochondrial gamma carbonic anhydrase reduces complex I and supercomplex I + III2 levels and alters mitochondrial physiology in Arabidopsis, J. Mol. Biol. 350 (2005) 263-277.

[55] K. Riazunnisa, L. Padmavathi, H. Bauwe, A.S. Raghavendra, Markedly low requirement of added $\mathrm{CO}_{2}$ for photosynthesis by mesophyll protoplasts of pea (Pisum sativum): possible roles of photorespiratory $\mathrm{CO}_{2}$ and carbonic anhydrase Physiol. Plant. 128 (2006) 763-772. 
[56] F. Villarreal, V. Martín, A. Colaneri, N. González-Schain, M. Perales, M. Martín, C. Lombardo, H-P. Braun, C. Bartoli, E. Zabaleta, Ectopic expression of mitochondrial gamma carbonic anhydrase 2 causes male sterility by anther indehiscence, Plant Mol. Biol. 70 (2009) 471-485.

[57] R. Sánchez, A. Flores, F.J. Cejudo, Arabidopsis phosphoenolpyruvate carboxylase genes encode immunologically unrelated polypeptides and are differentially expressed in response to drought and salt stress, Planta. 223 (2006) 901909.

[58] J. Schneidereit, E. Häusler, G. Fiene, W.M. Kaiser, A.P.M. Weber, Antisense repression reveals a crucial role of the plastidic 2-oxoglutarate/malate translocator DiT1 at the interface between carbon and nitrogen metabolism, Plant J. 45 (2006) 206-224.

[59] M.C. Wheeler, M.A. Tronconi, M.F. Drincovich, C.S. Andreo, U.I. Flügge, V.G. Maurino, A comprehensive analysis of the NADP-malic enzyme gene family of Arabidopsis, Plant Physiol. 139 (2005) 39-51.

[60] M.C. Wheeler, C.L. Arias, M.A. Tronconi, V.G. Maurino, C.S. Andreo, M.F. Drincovitch, Arabidopsis thaliana NADP malic enzyme isoforms: high degree of identity but clearly distinct properties. Plant Mol. Biol. 67 (2008) 231 242.

[61] P. Renné, U. Dreßen, U. Hebbeker, D. Hille, U.I. Flügge, P. Westhoff, A.P.M. Weber, The Arabidopsis mutant $d c t$ is deficient in the plastidic glutamate/malate translocator DiT2, Plant J. 35 (2003) 316-331.

[62] A. Bräutigam, S. Hoffmann-Benning, AP.M. Weber, Comparative proteomics of chloroplast envelopes from C3 and $\mathrm{C} 4$ plants reveals specific adaptations of the plastid envelope to $\mathrm{C} 4$ photosynthesis and candidate proteins required for maintaining C4 metabolite fluxes, Plant Physiol. 148 (2008) 568-579.

[63] A. Bräutigam, K. Kajala, J. Wullenweber, M. Sommer, D. Gagneul, K.L. Weber, K.M. Carr, U. Gowik, J. Mass, M.J. Lercher, P. Westhoff, J.M. Hibberd, A.P.M. Weber, An mRNA blueprint for C4 photosynthesis derived from comparative transcriptomics of closely related C3 and C4 species, Plant Physiol. 155 (2011) 142-156.

[64] Y. Taniguchi, J. Nagasaki, M. Kawasaki, H. Miyake, T. Sugiyama, M. Taniguchi, Differentiation of dicarboxylate transporters in mesophyll and bundle sheath chloroplasts of maize., Plant, Cell Physiol. 45 (2004) 187-200.

[65] N.J. Brown, C.A. Newell, S. Stanley, J.E. Chen, A.J. Perrin, K. Kajala, J.M. Hibberd, Independent and parallel recruitment of preexisting mechanisms underlying C4 photosynthesis, Science 331 (2011) 1436-1439.

[66] N.J. Brown, B.G. Palmer, S. Stanley, H. Hajaji, S.H. Janacek, H.M. Astley, K. Parsley, K. Kajala, W.P. Quick, S. Trenkamp, A.R. Fernie, V.G. Maurino, J.M. Hibberd, C4 acid decarboxylases required for C4 photosynthesis are active in the mid-vein of the $\mathrm{C} 3$ species Arabidopsis thaliana, and are important in sugar and amino acid metabolism, Plant $\mathrm{J}$. 61 (2010) 122-133.

[67] C. Dutilleul, S. Driscoll, G. Cornic, R. De Paepe, C.H. Foyer, G. Noctor, Functional mitochondrial complex I is required by tobacco leaves for optimal photosynthetic performance in photorespiratory conditions and during transients, Plant Physiol. 131 (2003) 264-275.

[68] G. Noctor, C. Dutilleul, R. De Paepe, C.H. Foyer Use of mitochondrial electron transport mutants to evaluate the effects of redox state on photosynthesis, stress tolerance and the integration of carbon/nitrogen metabolism. J. Exp. Bot. 55 (2004) 49-57.

[69] C.H Foyer, A.J. Bloom, G. Queval, G. Noctor, Photorespiratory metabolism: genes, mutants, energetics, and redox signaling, Annu. Rev. Plant. Biol. 60 (2009) 455-484.

[70] J.L. Heazlewood, J. Tonti-Filippini, R.E. Verboom, A.H. Millar, Combining experimental and predicted datasets for determination of the subcellular location of proteins in Arabidopsis, Plant Physiol. 139 (2005) 598-609.

[71] S. Reumann, L. Babujee, C. Ma, S. Wienkoop, T. Siemsen, G.E. Antonicelli, N. Rasche, F. Lüder, W. Weckwerth, O. Jahn, Proteome analysis of Arabidopsis leaf peroxisomes reveals novel targeting peptides, metabolic pathways, and defense mechanisms, Plant Cell 19 (2007) 3170-93. 
[72] C. Rode, M. Senkler, J. Klodmann, T. Winkelmann, H-P. Braun, GelMap-A novel software tool for building and presenting proteome reference maps. J. Proteomics. 74 (2011) 2214-2219.

[73] C.J. Ruan, H.B. Shao, J.A. Teixeira da Silva, A critical review on the improvement of photosynthetic carbon assimilation in C3 plants using genetic engineering. Crit. Rev. Biotech., (2011) 1-21 doi/10.3109/07388551.2010.533119.

[74] K. Kajala, S. Covshoff, S. Karki, H. Woodfield, B.J. Tolley, M.J. Dionora, R.T. Mogul, A.E. Mabilangan, F.R. Danila, J.M. Hibberd, W.P. Quick, Strategies for engineering a two-celled C(4) photosynthetic pathway into rice, J. Exp. Bot. 62 (2011) 3001-3010.

[75] K. Kajala, N.J. Brown, B.P. Williams, P. Borrill, L.E. Taylor, J.M Hibberd,. Multiple Arabidopsis genes primed for recruitment into C(4) photosynthesis. Plant J. (2011) doi: 10.1111/j.1365-313X.2011.04769.x.

[76] M. Miyao, C. Masumoto, S. Miyazawa, H. Fukayama, Lessons from engineering a single-cell C(4) photosynthetic pathway into rice, J.Exp Bot. 62 (2011) 3021-3029.

[77] G.D. Price, M.R. Badger, S. von Caemmerer, The prospect of using cyanobacterial bicarbonate transporters to improve leaf photosynthesis in C3 crop plants, Plant Physiol. 155 (2011) 20-26.

[78] R. Kebeish, M. Niessen, K. Thiruveedhi, R. Bari, H.J. Hirsch, R. Rosenkranz, N. Stäbler, B. Schönfeld, F. Kreuzaler, C. Peterhänsel, Chloroplastic photorespiratory bypass increases photosynthesis and biomass production in Arabidopsis thaliana. Nat Biotechnol. 25 (2007) 593-599.

[79] V.G. Maurino, U.I. Flügge, Means for improving agrobiological traits in a plant by providing a plant cell comprising in its chloroplasts enzymatic activities for converting glycolate into malate. Patent Application No. WO2009103782, 2009. 